\title{
Applying WIFI QoS Techniques to UMTS Networks
}

\author{
Belhocine Amin, Fedoua Didi \\ Department of Computer Science, Science Faculty, Abou Bekr Belkaid University, Tlemcen, Algeria \\ Email: belhocine_stic@yahoo.com,fedouadidi@yahoo.fr
}

Received May 24, 2013; revised June 25, 2013; accepted July 24, 2013

Copyright (C 2013 Belhocine Amin, Fedoua Didi. This is an open access article distributed under the Creative Commons Attribution License, which permits unrestricted use, distribution, and reproduction in any medium, provided the original work is properly cited.

\begin{abstract}
The last decade we have witnessed an unprecedented development of telecommunication technologies. This is not only due to devices capacity improvement, but also due to network performance amelioration. Moreover, new technologies were pro- posed such as UMTS (3G), giving users more mobility and therefore more freedom. However, this resulted in a more demanding application, particularly for QoS provisioning. WIFI and UMTS are among these new telecommunication technologies. Although each one has its own QoS techniques, WIFI experiences a lack in QoS provisioning and UMTS should be further improved to guaranty QoS. Our approach is simple. To be improved, each technology can benefit from QoS techniques of the other, of course, if this is possible. In this case, it's clear that UMTS can take advantage of WIFI QoS techniques, because UMTS uses physically a multitude of channels, using different bit rates and channel managements. While, WIFI uses a single channel with a single channel management, that is random access method. UMTS also uses a random access channel (PRACH channel), therefore we can apply WIFI QoS techniques to UMTS PRACH channel. In this study, we present WIFI and UMTS networks. We will focus on the air interface functioning of both networks to highlight similarities, then we propose two schemes inspired from WIFI QoS techniques to apply to UMTS air interface (Uu interface).
\end{abstract}

Keywords: Air Interface; QoS; UMTS; Uu Interface; WIFI

\section{Introduction}

Telecommunication technologies have become an essential part of the daily life of each of us. Among the most influencing are the wireless ones. More precisely, WIFI and cellular networks are the most used ones. WIFI has proved its efficiency through the last years, and UMTS, for the cellular phones, gained its place as the 3rd mobile generation around the world.

However, users today have a lot of expectations. They consider their equipment as a multimedia platform which provides different services. Those services (IP telephony, video streaming, etc.) involve different requirements to be satisfied by the network in order to guarantee them and to reach the user expectations.

QoS was created to guaranty these requirements. Its success in the computer science field is a big step forward that improves performances of all networks, especially in WIFI technology where radio resources are scarce and have to be exploited in a very good manner. Though, UMTS was designed with QoS in mind, we can still improve it by applying other technologies QoS techniques. In this study, we will outline the air interface similarities between WIFI and UMTS, and after we focus on applying WIFI QoS techniques to UMTS networks.

This paper is organized as follows: in Section 2 we present WIFI networks, in Section 3 we define QoS concept, in the next section we present WIFI QoS mechanisms. Section 5 describes UMTS network elements and particularly those related to the air interface, the next section presents our approach where we describe two new schemes, and finally Section 8 concludes the paper.

\section{WIFI (802.11)}

802.11 is part of IEEE 802 family. As any other 802.x protocol, the 802.11 protocol covers the MAC sub-layer and the physical layer. This standard defines only one MAC sublayer that interacts with three physical layers:

- Frequency Hopping spread spectrum in the $2.4 \mathrm{GHz}$ band.

- Direct Sequence spread spectrum in the $2.4 \mathrm{GHz}$ band.

- Infrared.

\subsection{Medium Access Method}

The DCF (Distributed Coordination Function) is a fun- 
damental access method that stations have to support. It supports an asynchronous transfer of data based on best effort. The DCF is based on contention principal; it means that all stations access the same transmission medium. Physically, it's based on CSMA/CA (Carrier Sense Multiple Access with Collision Avoidance). The carrier detection (carrier sense) implies that all stations monitor the medium to determine if it is busy or not [1]. The PCF (Point Coordination Function) is an optional access method and is limited to infrastructure based networks.

It supports delay sensitive transfer data (voice, video, etc.). The PCF allows transmission on the medium without contention. The PC (Point Coordinator) controls all the traffic by polling all the stations. After being polled only, a station can transmit a frame.

When PCF and DCF coexist, the transmission capacity is shared between traffic without contention during a contention free period (CFP) and traffic with contention during a contention period (CP). The alternation of contention periods and contention free periods is repeated at regular interval called repeat interval [1].

\subsection{Binary Exponential Backoff Algorithm}

The backoff is a well-known method to solve the access problem between many stations looking for access to medium.

In this method, every station chooses a random waiting delay between 0 and a contention window size (CW) that is equal to a number of slots, and waits this number of slots before transmitting, and always making sure that another station has not accessed the medium before it.

A slot time is defined in such a way that the station will always be capable to determine if another station has accessed the medium at the beginning of the previous slot. This divided the collision probability by two. The exponential backoff means that every time a station chooses a random waiting delay and causes a collision, the random waiting delay is exponentially increased (doubled) in the following attempted transmission. The only case where this mechanism isn't used is when the station decides to transmit a new packet and the medium was free during a period longer than DIFS. The random waiting duration (RWD) of backoff is calculated in the following way:

$$
\mathrm{RWD}=\mathrm{CW} \cdot \text { random }(0, \mathrm{CW}) \cdot \text { SlotTime }
$$

where random $(0, \mathrm{CW})$ is a uniform random variable included between 0 and CW-1, CW is the size of the contention window, $\mathrm{CW}=\left[\mathrm{CW}_{\min } \mathrm{CW}_{\max }\right]$, in the first transmission attempt $\mathrm{CW}=\mathrm{CW}_{\min }$, and in the following attempt (in a collision case) CW is doubled until it reaches $\mathrm{CW}_{\max }$.

\section{QoS}

The QoS is the fact to foster the transition of a specific traffic type compared to others, by reserving network resources somehow directly or indirectly.

QoS is the ability of a network element to ensure that its traffic and service needs can be met. QoS is often accomplished by providing different prioritizations for different type of traffic in a network. According to international telecommunication union (IUT), QoS is the collective effect of services performance, which determines the satisfaction degree of a service user. The internet engineering task force (IETF) gives an other definition of QoS. Its approach is that the QoS is a set of needs of services that must be met by the network when it carries a stream. The goal of a communication system that guarantees QoS is the effective use of bandwidth, delay limits and jitter, acceptable error rate, and adaptability to dynamic changes of network and traffic conditions. It offers earnings to voice on WLAN, real time communications, and radio and video streams [2].

\section{QoS for WIFI}

In order to offer limited time services the 802.11a defined the PCF to allow stations to get priority in the medium access procedure, coordinated by a station called point coordinator (PC) [2]. This was the first attempt to reach a QoS in WIFI. PCF has higher priority than DCF as the PC waits for a PIFS period which is shorter than DIFS in DCF.

\subsection{QoS Limits in IEEE 802.11 Standard}

DCF supports only best effort services and provides no guarantee of QoS to multimedia applications. There is no provision to support QoS in DCF because all traffic is treated with the first in first out mode.

Although PCF is designed by IEEE group to support limited-time multimedia applications, this mode has some major problems that lead to a poor QoS. In particular, the central polling scheme is inefficient and complex and causes the degradation of the performance of high-priority PCF traffic under load [2]. Additionally, all communications must go through AP (Access Point), which degrades the performance of the bandwidth $[3,4]$.

\subsection{The 802.11e Standard}

The 802.11e standard defines one coordination function called hybrid coordination function (HCF). The HCF is composed of two channel access mechanisms:

- A channel access based on contention called enhanced distributed channel access (EDCA).

- A controlled channel access called HCF controlled channel access (HCCA).

EDCA is an improvement of DCF, and HCCA is an improvement of PCF [2].

One of the distinctive characteristics of 802.11e is the 
opportunity transmission concept (TXOP), which is an interval of time in which a particular station has the right to initiate transmissions. The new concept in TXOP is the limitation of the time interval in which a station can transmit its frames.

\subsubsection{Enhanced Distributed Channel Access}

EDCA is designed to provide differentiated and distributed channel access for packets with eight different priorities (from 0 to 7) improving DCF.

Each frame arriving to the MAC with a user priority corresponds to an access category (AC) as illustrated in Table 1, where an access function is used for each AC [5].

A channel access function uses AIFS [AC], $\mathrm{CW}_{\text {min }}$ [AC], $\mathrm{CW}_{\max }$ [AC] instead of DIFS, $\mathrm{CW}_{\min }, \mathrm{CW}_{\max }$ in DCF, respectively, for the contention process to transmit a packet belonging to an access category (AC). AIFS [AC] is defined as follows:

$$
\text { AIFS }[\text { AC }]=\text { SIFS + AIFSN }[\text { AC }] . \text { SlotTime }
$$

where AIFSN [AC] is an integer greater than 1 for nonAP QSTA stations and an integer greater than 0 for QAP. The backoff counter is selected from [0, CW [AC]]. Figure 1 illustrates the EDCA temporal diagram [5].

During an EDCA TXOP, as illustrated in Figure 2, there may be multiple frame exchange sequences, separated by SIFS, and initiated by a single station (or more precisely, by a station EDCAF). A TXOP can be obtained

Table 1. User Priority to Access Category Mappings [5].

\begin{tabular}{ccc}
\hline User Priority & Access Gategory (AC) & Designation (Informative) \\
\hline 1 & AC_BK & Background \\
2 & AC_BK & Background \\
0 & AC_BE & Best effort \\
3 & AC_BE & Best effort \\
5 & AC_VI & Video \\
6 & AC_VI & Video \\
7 & AC_VO & Voice \\
\hline
\end{tabular}

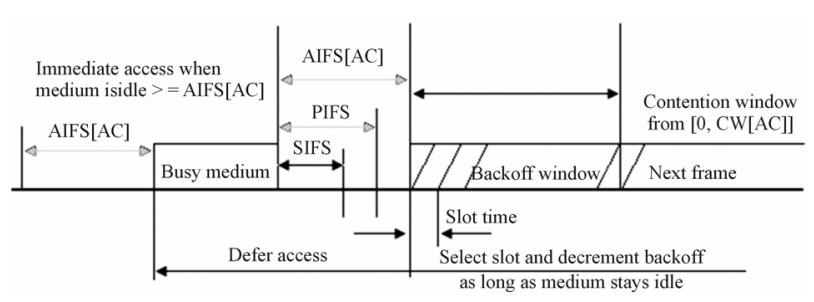

Figure 1. The channel access scheme for IEEE 802.11e EDCA [5].

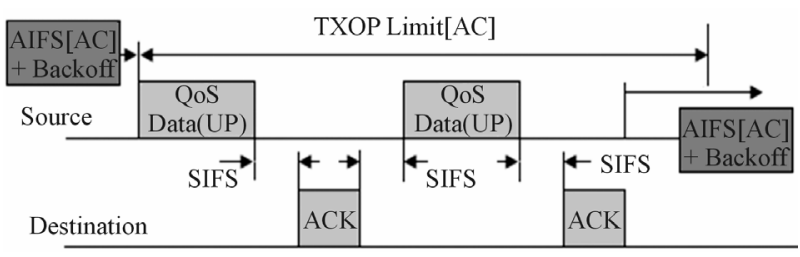

Figure 2. The temporal working structure of EDCA TXOP [5].

by a successful EDCA contention of the corresponding EDCAF. The duration of a TXOP is determined by an other EDCA parameter called TXOP limit. This value is defined for each AC, and therefore is called TXOP-Limit [AC].

\subsubsection{HCF Controlled Channel Access}

HCCA is an extension of PCF. As in PCF, with HCCA, the superframe is divided into CFP and CP. The CFP starts with each beacon frame and the access is governed by EDCA during the CP, however the HC (Hybrid Coordinator) can initiate HCCA access at any time [4]. The HC sends a QoS CF-Poll to a particular station to give it a TXOP, by specifying the beginning time and the maximum duration. No station attempt to access the medium at that time, and therefore when a station receives a CFPoll, it takes a TXOP and transmits any data that is in its possession. The beacon frame or a CF-End frame determines the end time of a CFP. A station must begin transmission with data in a period of SIFS if it receives a CF-Poll frame. After PIFS, the HC can take the control of the medium and transmit a CF-Poll to another station if the previous station doesn't begin the transmission. The medium utilization is very efficient during a CFP.

If the base station wishes to initiate a transmission sequence in CP, the HCCA allows it to do so. The HC has a snapshot view on the queue length information per station and per TC in the cell including that of AP sent by stations via the new field of QoS control added to the MAC frame. This information is important for the HC to decide to which station (including itself) allocate the TXOP during the CFP. To allocate TXOP, the HC considers TC priority, the required QoS for the TC, the length of the queue per TC, the length of the queue per station, the available duration and to allocate of TXOP, and the QoS experienced in the past by the TC [2]. The HC needs information that must be updated by the polled stations from time to time. The HC maintains a path called controlled contention to learn which stations need to be polled, when, and for how long [2].

\section{UMTS Networks}

A UMTS network consists of logical entities each providing a specific function. These entities are grouped into UTRAN (UMTS Terrestrial Radio Access Network) re- 
sponsible for radio functions, UE (User Equipment) exploiting the radio medium and the CN (Core Network) responsible for commutation and routing calls and data to external networks.

In this study, we focus on the air interface architecture to highlight similarities with the air interface of WIFI technology. The architecture of radio interface protocol $(\mathrm{Uu})$ is organized into three layers : network layer (layer 3 ), link layer (layer 2) and physical layer (layer 1). The link layer is divided into 4 sub-layers: MAC (Medium Access Control), RLC (Radio Link Control), BMC (Broadcast Multicast Control), and PDCP (Packet Data Convergence Protocol) as shown in the following Figure 3. We will focus on physical and MAC layers.

Layer 3 contains RRC that treats the control plan sig-naling from UTRAN to UE, and is responsible for the configuration and control of all the other layers of UTRAN protocol [6,7].
The physical layer provides services to the MAC layer through transport channels. These channels are specified according to characteristics of data to transmit and the way to transmit them. The MAC layer provides services to the RLC layer by means of logical channels characterizing the data type to be transmitted.

\subsection{Physical Layer}

The physical layer is based on WCDMA technology, and Figure 4. Radio Interface protocol architecture. The Service Access Points are marked by circles $[7,8]$ utilizes many physical channels. In UTRAN, data from upper layers are transmitted by air with transport channels, which are mapped in the physical level to many physical channels. The physical layer is required to support transport channels with variable bit rates ensuring the bandwidth services on demand and the multiplexing of

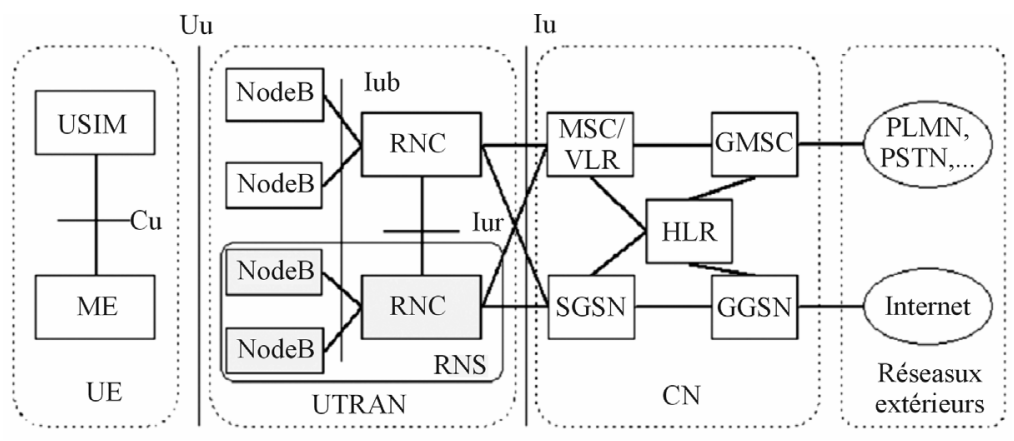

Figure 3. UMTS network architecture.

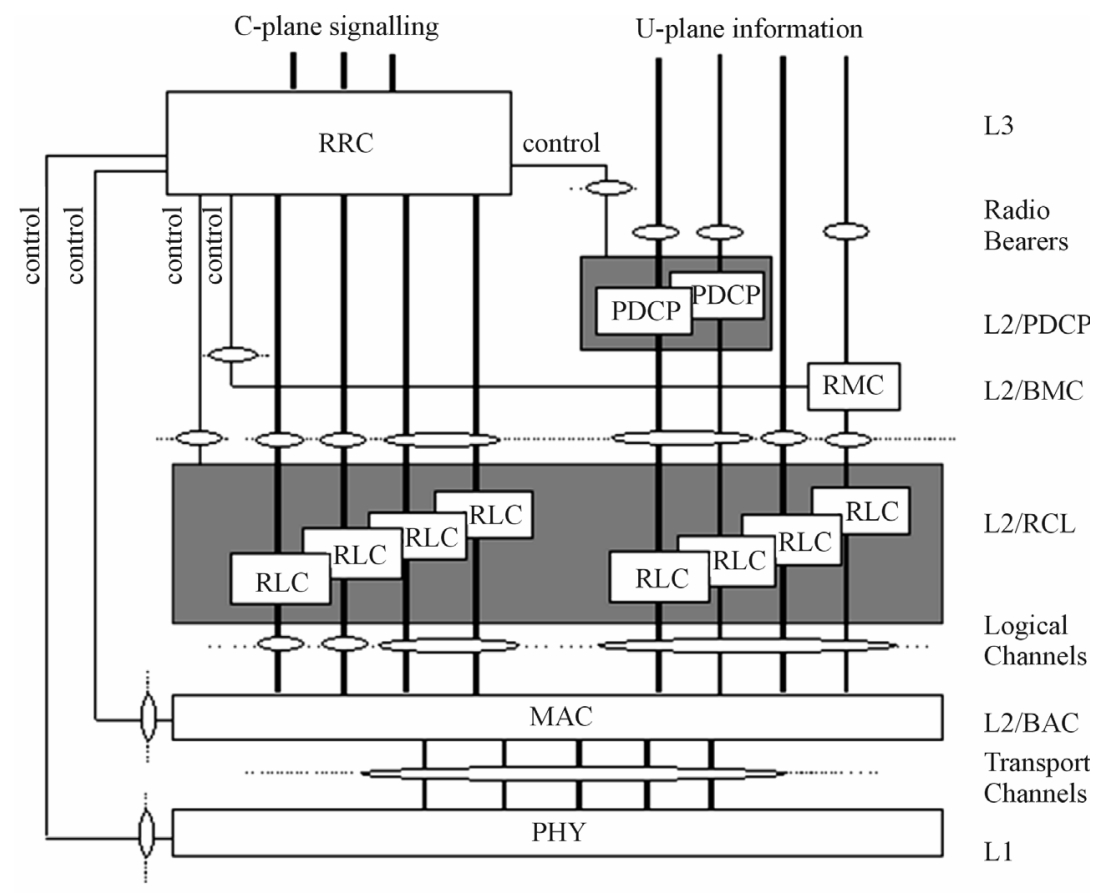

Figure 4. Radio interface protocol architecture. The service access points are marked by circles $[7,8]$. 
services on a connexion [9].

\subsection{MAC Protocol}

In the MAC layer, logical channels are mapped to transport channels. The MAC layer is also responsible for selecting the transport format (TF) suitable for each transport channel according to the throughput of logical channels. The MAC layer is informed of the allocations of resources by the RRC. The priority treatment between different data flows, which are mapped to the same physical resources, is also realized by the MAC layer [7].

Figure 5 illustrates the mapping between logical and transport channels in the MAC layer seen from the UE side.

As illustrated in this figure, the MAC layer uses many transport channels. As we are looking for similarities between the air interfaces working of WIFI and UMTS, we will describe the RACH channel functioning because it's based on contention access, that is the access principal used in WIFI. Therefore RACH presents the channel on which we can apply the WIFI QoS mechanisms and parameters.

\section{Random Access Channel (RACH)}

PRACH (Physical RACH) is a physical channel mainly used to transport control data (random access requests) from UE (User Equipment) to BS (Base Stations). The random access is used by UE for the initial access, requesting dedicated channels and transmitting short data packets to BS $[10,11]$.

UTRAN uses two physical modes to transmit its frames; FDD (Frequency Division Duplex) mode and TDD (Time Division Duplex) mode. The TDD frame is composed of 15 time slots per radio frame of $10 \mathrm{~ms}$ duration on a single frequency with $5 \mathrm{MHz}$ bandwidth, thus 240 RU (Radio Units) can be considered as illustrated in Figure 6. The TDD frame is divided into downlink and uplink parts. The switching point between uplink and downlink can be moved in the TDD frame to support asymmetric traffic $[12,13]$. The QoS mechanism we propose can be applied on the TDD frame, considering random accesses provided by the uplink part of the frame.

In FDD mode, RACH is composed of eight times slots (opportunities) in which we can initiate transmissions. In each time slot, RACH can transmit until 16 frames in the same time because UMTS air interface is based on CDMA (Code Division Multitude Access). CDMA allows the transmission of multiple frames, one frame by code (until 16 codes or signatures). Each time slot has duration of $1.25 \mathrm{~ms}$, thus $1.25 \times 8=10 \mathrm{~ms}$ which represents UMTS frame duration. Therefore, RACH can transmit until $8 \times 16=128$ frames in a maximum configuration, as shown in Figures 7 and 8 [15].

Figure 8 illustrates access slots in random access channel with examples of frames using these accesses.

\subsection{UE Connection Procedure (Access Method to the Medium)}

When a user needs a service from the network, its user equipment (UE) sends a channel request to the network through the physical random access channel (PRACH), a specific channel dedicated to the uplink transmission of channel request. Since the network does not control the PRACH usage, the access method, based on a random access procedure, may cause collisions among requests by different UEs, thus worsening the expected QoS.

\section{Related Work}

To our best knowledge, very few research works have been done to improve QoS in Uu interface and even less to apply QoS techniques inspired from WIFI to UMTS air interface. For example, in [15] the authors proposed three schemes to provide access priority in the RACH transport channel. These schemes are: random chip delay access priority (RCDAP), random backoff based

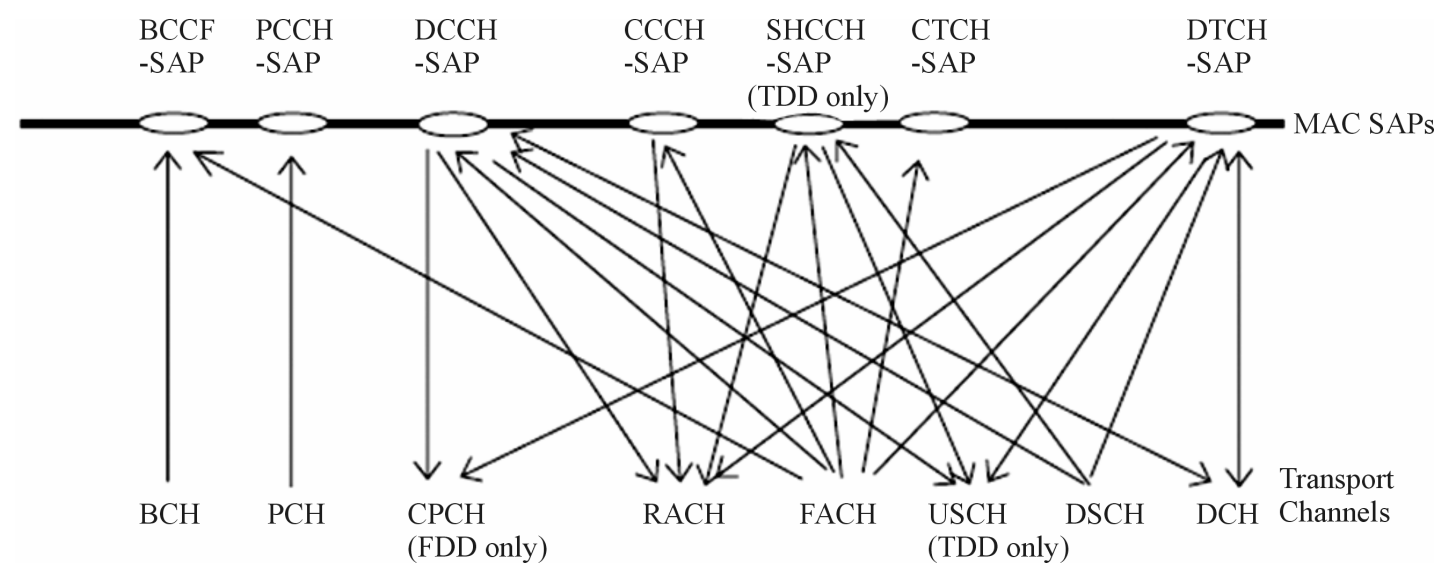

Figure 5. Logical channels mapped onto transport channels, seen from the UE side [8]. 


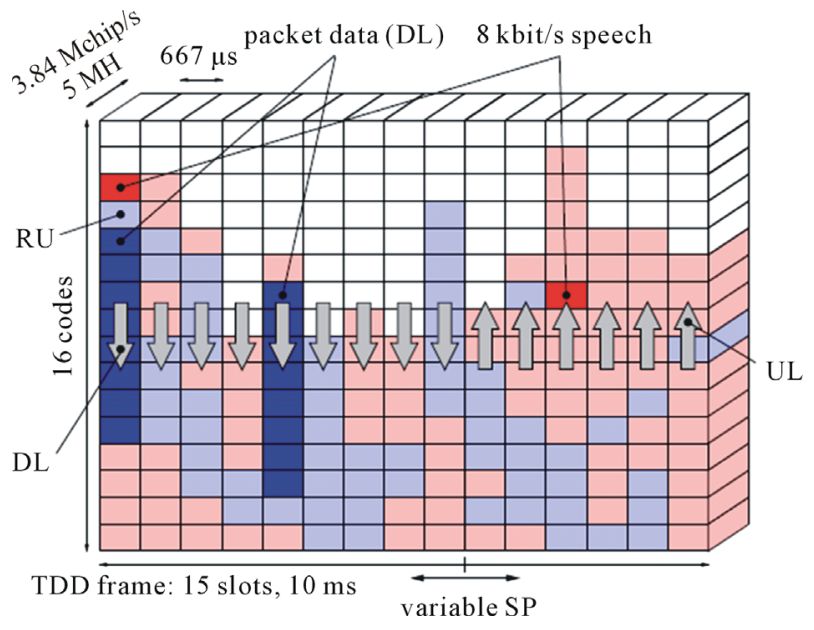

Figure 6. Radio frame configuration in UTRA-TDD with asymmetric time slot [12,13].

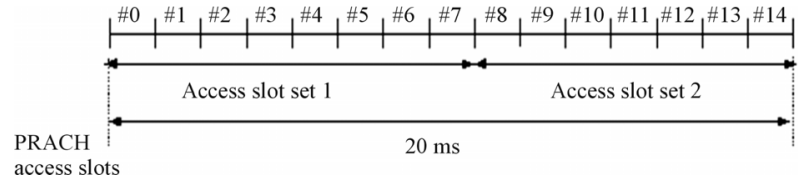

Figure 7. PRACH access slot [14].

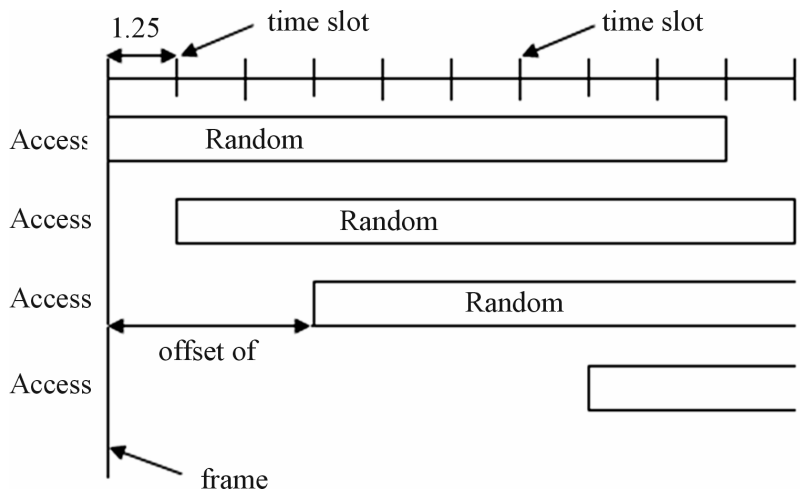

Figure 8. Access slots in PRACH [15].

access priority (RBBAP) and variable logical channel based access priority (VLCAP). RBBAP scheme utilizes backoff delay to differentiate between prioritized classes (traffics) and less prioritized classes like with the $\mathrm{Cw}_{\min }[\mathrm{AC}]$ and $\mathrm{Cw}_{\max }[\mathrm{AC}]$ parameters in EDCA QoS mechanism in WIFI. This scheme can be seen as applying a WIFI QoS technique to improve QoS in UMTS air interface, even if it's not explicitly mentioned by the authors.

\section{QoS in Umts Air Interface (our Approach)}

In UMTS the access system is an infrastructure system and the priority schemes (access) will be handled or managed from RRC protocol. To reach the user satis- faction, the network has to guarantee end to end QoS. Among all the UMTS network elements, the air interface (Uu interface) is the component where resources are the most scarce and therefore constitutes a bottleneck in the system. This means that QoS in Uu interface must be improved.

To improve QoS in Uu interface, we propose two mechanisms inspired from QoS handling in WIFI networks.

The first is ORAP (Ordred Random Access Priority) scheme and the second is TOP (Transmit Opportunity) scheme. Both of them are based on RACH channel.

\subsection{ORAP (Ordred Random Access Priority) Scheme}

Random access (RACH) offers eight (8) access opportunities (with 16 codes for each opportunity) to transmit a UMTS frame. In ORAP scheme, each access opportunity (AO) or access slot is dedicated to a higher priority frame than the following access opportunity. That means that the first $\mathrm{AO}$ is dedicated to the highest priority frames (AO [P1]), the second $\mathrm{AO}$ is dedicated to lower priority frames, etc... and the last $\mathrm{AO}$ is dedicated to the lowest priority frames. So the order of Access Opportunities corresponds to the order of frames priorities (from the highest to the lowest). Thus this mechanism offers 16 opportunities to transmit frames of the highest priority in the first AO. This mechanism is inspired from AIFS [AC] QoS parameter of EDCA in WIFI networks as illustrated in Figure 9.

For example, an audio traffic having the User Priority 7 in WIFI (Table 1) and thus being the most prioritized traffic in the network (AIFS [AC_VO]), will be affected to P1 class in ORAP scheme. Therefore, this audio traffic will get the most highest priority in our ORAP scheme, and will access the PRACH channel using the parameter AO [P1]. This mechanism ensures to the audio traffic to get access to the channel before any other traffic less

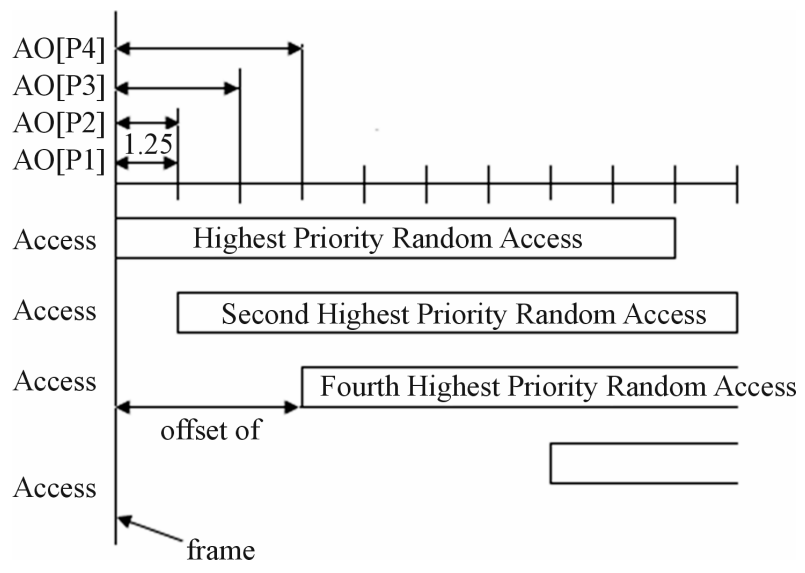

Figure 9. Ordred random access priority scheme. 
prioritized such as P2 for example.

\subsection{TOP (Transmit Opportunity) Scheme}

To give a UE (User Equipment) priority to transmit its frame, TOP scheme gives it a set of access slots dedicated to it. For example, if TOP scheme provides the UE 4 access slots, the UE will have $4 \times 16=64$ opportunities to transmit its frame using random access channel. This limit (4 in our example) is defined by TOPLimit [P] parameter. During this period, no other UE has the permission to transmit. This mechanism uses the same principle as in TXOP (transmit opportunity) QoS parameter of HCCA used in WIFI, and TOPLimit [P] is equivalent to TXOPLimit[AC] parameter in HCCA. Figure 10 shows TOP scheme.

For example, the same audio traffic with the User Priority 7 in WIFI, will be provided 3 access slots (for example) by the TOP scheme. Thus, during these 3 access slots, no other traffic has the right to access the PRACH channel, and therefore, this audio traffic will have $3 * 16$ $=48$ access opportunities to initiate a transmission over PRACH channel. The number of access slots (here 3 ) is defined by the TOPLimit [P1] parameter.

\section{Conclusion}

In this study, we address WIFI and UMTS networks and their handling to QoS in the air interface. WIFI is based on contention, while UMTS utilizes multiple channels including a contention based channel (RACH). As this channel is also based on contention, we propose two schemes inspired from QoS handling in WIFI to improve QoS in UMTS air interface.

The first is ORAP scheme and is based on AIFS [AC] QoS parameter used in WIFI, and the second is TOP scheme and is based on TXOP QoS parameter also used in WIFI. These schemes will improve QoS handling in UMTS air interface and therefore contribute in getting an enhanced end to end QoS and involve a better user

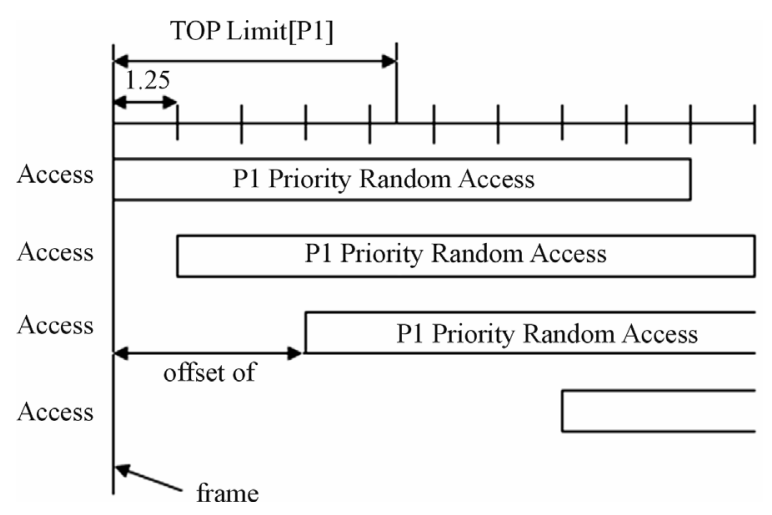

Figure 10. Transmit opportunity scheme. experience and satisfaction.

In the future, our goal is to simulate and validate our propositions. This will be done by comparing our schemes with those proposed in [15].

\section{REFERENCES}

[1] "IEEE Standard for Information Technology-Telecommunications and Information Exchange between SystemsLocal and Metropolitan Area Networks-Specific Requirements-Part 11: Wireless LAN Medium Access Control (MAC) and Physical Layer (PHY) Specifications,” 1997.

[2] A. Based, “A Survey about IEEE 802.11e for Better QoS in WLANs," Proceedings of the International Conference of Novel Algorithms and Techniques in Telecommunications and Networking, 2010, pp. 195-200.

[3] M. Ma, M. K. Denko and Y. Zhang, "Wireless Quality of Service: Techniques, Standards, and Applications,” CRC Press, Boca Raton, 2009.

[4] D. Fedoua, "Qualité de Service dans les réseaux locaux sans fil de type IEEE 802.11,” Ph.D. Thesis, Abou Bekr Belkaid University, Tlemcen, 2010.

[5] R. Shorey, A. Ananda, M. C. Chan and W. T. Ooi, "Mobile, Wireless, and Sensor Networks," Wiley-Interscience, New York, 2006.

[6] A. Andreadis and G. Giambene, "Protocols for High-Efficiency Wireless Networks,” Part II, Springer, Berlin, 2002, pp. 175-204.

[7] C. Gessner, R. Kohn, J. Schniedenharn and A. Sitte, "UTRA TDD Protocol Operation," Proceedings of The 11th IEEE International Symposium on Personal, Indoor and Mobile Radio Communications, Vol. 2, London, 2000, pp. 1226-1230.

[8] 3GPP, “Radio Interface Protocol Architecture,” 2002.

[9] 3GPP, "Physical Layer-General Description. 1999. http://www.3gpp.org/ftp/specs/archive/25_series/25.201/

[10] Y. Yang and T. S. P. Yum, "Analysis of Power Ramping Schemes for UTRA-FDD Random Access Channel," Proceedings of IEEE Global Telecommunications Conference, Vol. 5, Beijing, 2004, pp. 3305-3309.

[11] W.-H. Sheen, C.-C. Tseng and J.-S. Ho, "Burst Synchronization of Slotted Random Access With Preamble Power Ramping in the Reverse Link of CDMA Systems," IEEE Transactions on Wireless Communications Conference, Vol. 2, No. 5, 2003, pp. 953-963. doi:10.1109/9.402235

[12] I. Forkel, B. Wegmann and E. Schulz, "On the Capacity of a UTRA-TDD Network with Multiple Services," Proceedings of ICC 2002 IEEE International Conference, Vol. 1, 2002, pp. 585-589.

[13] C. Mihailescu, X. Lagrange and P. Godlewski, "Performance Evaluation of a Dynamic Resource Allocation Algorithm for UMTS-TDD Systems," Proceedings of Vehicular Technology Conference, Vol. 3, Tokyo, 2000, pp. 2339-2343.

[14] 3GPP, "Physical layer procedures (FDD) (Release 10)," 2011. 
[15] M. Chuah, Q. Zhang and O. Yue, “Access Priority Schemes in UMTS MAC," Proceedings of IEEE Wireless 2003, pp. 781-786. Communications and Networking Conference, Vol. 2, 